\title{
A GREEDOID POLYNOMIAL WHICH DISTINGUISHES ROOTED ARBORESCENCES
}

\author{
GARY GORDON AND ELIZABETH MCMAHON
}

(Communicated by Thomas $\mathrm{H}$. Brylawski)

\begin{abstract}
We define a two-variable polynomial $f_{G}(t, z)$ for a greedoid $G$ which generalizes the standard one-variable greedoid polynomial $\lambda_{G}(t)$. Several greedoid invariants (including the number of feasible sets, bases, and spanning sets) are easily shown to be evaluations of $f_{G}(t, z)$. We prove (Theorem 2.8) that when $G$ is a rooted directed arborescence, $f_{G}(t, z)$ completely determines the arborescence. We also show the polynomial is irreducible over $\mathbf{Z}[t, z]$ for arborescences with only one edge directed out of the distinguished vertex. When $G$ is a matroid, $f_{G}(t, z)$ coincides with the Tutte polynomial. We also give an example to show Theorem 2.8 fails for full greedoids. This example also shows $f_{G}(t, z)$ does not distinguish rooted arborescences among the class of all greedoids.
\end{abstract}

\section{INTRODUCTION}

In this paper, we define a two-variable polynomial $f_{G}(t, z)$ for a greedoid $G$ which generalizes the one-variable polynomial $\lambda_{G}(t)$ given in [2, Section 9.6]. The main theorem states that when $G$ is a rooted directed arborescence, $f_{G}(t, z)$ completely determines the arborescence. All the graphs in this paper are finite. In this section, we recall some definitions from graph theory and define the polynomial. A more complete account of the graph theory can be found in [7], for example.

Definition 1.1. Let $D=(V(D), E(D))$ be a rooted directed graph (rooted digraph), i.e., a directed graph with a distinguished vertex, denoted $(*) .(V(D)$ and $E(D)$ represent the vertices and directed edges of $D$, respectively.) A rooted subdigraph $F$ is a rooted arborescence if the root vertex $*$ is in $F$ and, for every vertex $v$ in $F$, there is a unique directed path in $F$ from $*$ to $v$. Thus, rooted arborescences in digraphs correspond to rooted trees in undirected graphs. See Figure 1 for an example.

Received by the editors August 12, 1988 and, in revised form, November 4, 1988.

1980 Mathematics Subject Classification (1985 Revision). Primary 05C20, 05 B35.

Key words and phrases. Greedoid, rooted digraph, arborescence.

The work of the first author was partially supported by Lafayette College CASR summer research grant. 


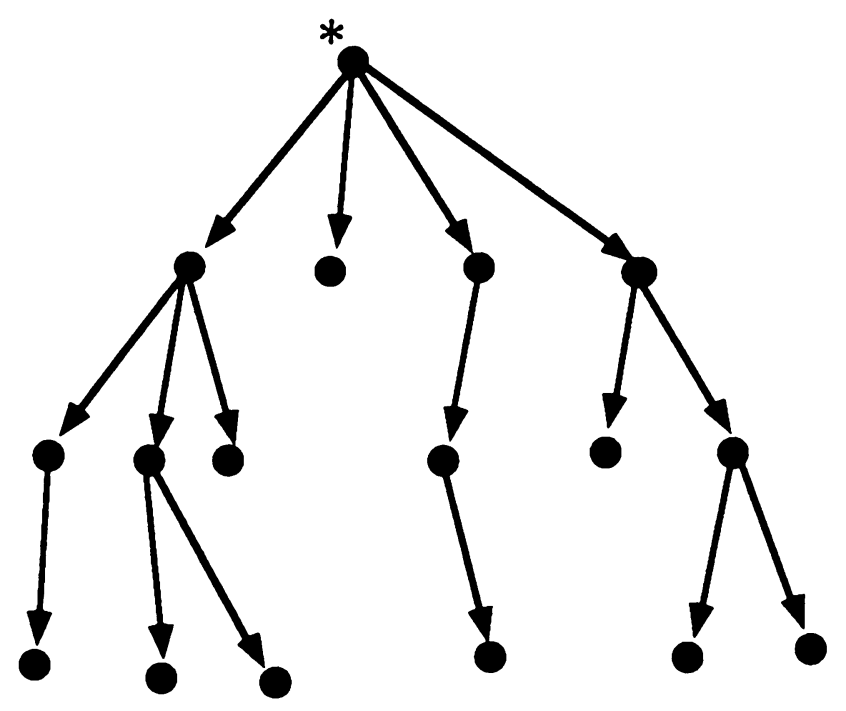

Figure 1

Definition 1.2. A subdigraph $F$ of a rooted digraph $D$ is said to be feasible if $F$ is a rooted arborescence. We will also call the edges of $F$ a feasible set of edges, or simply a feasible set, when no confusion can arise. $B \subseteq E(D)$ is called a basis if $B$ is a maximal feasible set. It is a standard fact that all bases have the same cardinality. $S \subseteq E(D)$ is a spanning set if $S$ contains a basis. For any $A \subseteq E(D)$, we define the rank of $A$, denoted $r(A)$, as follows: $r(A)=\max \{|F|: F \subseteq A, F$ is feasible $\}$.

For any $A, B \subseteq E(D)$ and any $x, y \in E(D)$, the rank function satisfies the following:

R1. $r(A) \leq|A|$.

R2. If $A \subseteq B$, then $r(A) \leq r(B)$.

R3. If $r(A)=r(A \cup\{x\})=r(A \cup\{y\})$, then $r(A)=r(A \cup\{x, y\})$.

Several remarks are in order here. Any function $r: 2^{E} \rightarrow \mathbf{N}$ satisfying properties R1-R3 defines a greedoid $G$ on $E$. Then a set $F$ is defined to be feasible iff $r(F)=|F|$. Greedoids can also be defined by specifying the collection of feasible sets. Bases and spanning sets are defined exactly as above, and it is again a standard exercise to show that all bases are equicardinal. In this context, rooted digraphs are termed directed branching greedoids. The rank function defined above is called the independence rank. It is also easy to construct examples of greedoids which are not directed branching greedoids. An extensive introduction to greedoids can be found in [2]. Many earlier papers by Korte and Lovasz form the basis for [2]—see [6], for example.

We now define a two-variable greedoid polynomial. 
Definition 1.3. Let $G$ be a greedoid, with rank function as defined above. Define $f_{G}(t, z)$ as follows:

$$
f_{G}(t, z)=\sum_{A \subseteq G} t^{r(G)-r(A)} z^{|A|-r(A)} .
$$

In what follows, when confusion cannot result, we will occasionally write $f_{G}$, $f(G)$, or $f(t, z)$ for $f_{G}(t, z)$ for ease of notation.

The definition of $f_{G}$ is motivated by the Tutte polynomial of a matroid (or, more precisely, the corank-nullity version of the Tutte polynomial). A matroid can be defined as a greedoid whose rank function satisfies R2' $: r(A) \leq r(A \cup$ $\{x\}) \leq r(A)+1$ (unit-rank increase). (Note that R2' implies R2.) The feasible sets of a matroid are called independent sets. General information about matroids can be found in [3] or [8], for example. One treatment of the Tutte polynomial concentrating on applications to graphs and codes can be found in [4].

We also note that other definitions of rank in greedoids or rooted digraphs give rise to corresponding polynomials via (1.3). For example, the basis rank in a greedoid, denoted $\beta(A)$, is defined by $\beta(A)=\max \{|B \cap A|: B$ is a basis $\}$. We remark that using $\beta(A)$ in (1.3) gives a polynomial which does not distinguish rooted arborescences, i.e., Theorem 2.8 below is not true for this polynomial. In fact, this polynomial is the same for any rooted arborescence on $n$ edges. We do not explore the various possibilities and interrelations here; see [1] and [5] for details.

In Section 2, we concentrate on rooted digraphs, proving the main theorem concerning rooted arborescences, namely, that if $T_{1}$ and $T_{2}$ are both rooted arborescences with $f\left(T_{1}\right)=f\left(T_{2}\right)$, then $T_{1}$ and $T_{2}$ are isomorphic rooted digraphs. We also give some results concerning the reducibility of $f_{T}(t, z)$ over $\mathbf{Z}[t, z]$, when $T$ is a rooted arborescence. In Section 3, we discuss the generalizations to greedoids and matroids and give some counterexamples. In particular, we show the polynomial $f_{G}(t, z)$ does not distinguish the class of rooted arborescences, i.e., we give an example where $f_{G}(t, z)=f_{D}(t, z)$ with $D$ a rooted arborescence and $G$ a greedoid which is not a directed branching greedoid.

\section{ROOTED DIGRAPHS}

We begin with some properties of the polynomial defined in Section 1.

The statements in the following lemma are easy to establish.

\section{Lemma 2.1. Let $D$ be a rooted digraph.}
a. The coefficient of $t^{r(D)-1}$ is the outdegree of *.
b. $f_{D}(1,1)=2^{|E(D)|}$.
c. $f_{D}(1,0)=$ the number of feasible sets.
d. $f_{D}(0,1)=$ the number of spanning sets.
e. $f_{D}(0,0)=$ the number of bases.
f. If $D$ is an arborescence, then for any term $c t^{a} z^{b}$ in $f_{D}, a \geq b$. Further, $a=b$ implies $a=b=0$. 
The next lemma gives several ways to check whether $D$ is a rooted arborescence from the polynomial $f_{D}(t, z)$.

Lemma 2.2. The following are equivalent:

a. $D$ is an arborescence.

b. $f_{D}(0,1)=1$.

c. In every term the $z$ exponent $\leq$ the $t$ exponent.

$d$. There is no pure $z$ term.

Proof. $a \Leftrightarrow b . D$ is an arborescence if and only if the number of spanning sets is 1 , or, by Lemma 2.1.d, $f_{D}(0,1)=1$.

$a \Rightarrow c$. If $D$ is an arborescence, then $r(D)=|E(D)| \geq|A| \geq r(A)$ for any subset $A$ of edges of $D$. Hence, $r(D)-r(A) \geq|A|-r(A)$.

$c \Rightarrow d$. Obvious.

$d \Rightarrow a$. If there is no pure $z$ term, then for every subset of edges $A, r(D)=$ $r(A)$ implies $|A|=r(A)$. In particular, then, $|E(D)|=r(D)$. Thus, $D$ itself is a feasible set, so $D$ is an arborescence.

We recall the definition of the direct sum of rooted digraphs. We note that our formulation of this definition will essentially be an internal direct sum; the external direct sum can be defined similarly.

Definition 2.3. Let $D_{1}$ and $D_{2}$ be subsets of edges of $D$. Then $D$ is the direct sum of $D_{1}$ and $D_{2}$, written $D=D_{1} \oplus D_{2}$, if $D_{1} \cap D_{2}=\varnothing, D_{1} \cup D_{2}=D$, and the feasible sets of $D$ are precisely the unions of feasible sets of $D_{1}$ and $D_{2}$.

Proposition 2.4. If $D=D_{1} \oplus D_{2}$, then $f(D)=f\left(D_{1}\right) \cdot f\left(D_{2}\right)$.

Proof. Let $F$ be the set of feasible sets in $D$, and $F_{1}$ and $F_{2}$ those in $D_{1}$ and $D_{2}$, respectively. Then $F=\left\{\mathbf{F} \cup \mathbf{F}^{\prime}: \mathbf{F} \in F_{1}, \mathbf{F}^{\prime} \in F_{2}\right\}$. Note first that by the definition of direct sum, there exist bases $B_{1}$ in $F_{1}$ and $B_{2}$ in $F_{2}$ such that $r(D)=\left|B_{1}\right|+\left|B_{2}\right|=r\left(D_{1}\right)+r\left(D_{2}\right)$. Now let $A$ be a subset of edges of $D$. Since $A=A_{1} \cup A_{2}$, with $A_{1}$ in $D_{1}$ and $A_{2}$ in $D_{2}$, then there is $\mathbf{F}_{1} \in F_{1}, \mathbf{F}_{2} \in F_{2}$ with $\mathbf{F}_{1} \subseteq A_{1}, \mathbf{F}_{2} \subseteq A_{2}$ such that $r(A)=\left|\mathbf{F}_{1}\right|+\left|\mathbf{F}_{2}\right|=r\left(A_{1}\right)+r\left(A_{2}\right)$. Thus,

$$
t^{r(D)-r(A)} z^{|A|-r(A)}=\left[t^{r\left(D_{1}\right)-r\left(A_{1}\right)} z^{\left|A_{1}\right|-r\left(A_{1}\right)}\right]\left[t^{r\left(D_{2}\right)-r\left(A_{2}\right)} z^{\left|A_{2}\right|-r\left(A_{2}\right)}\right] .
$$

Finally, since every subset $A$ of edges of $D$ splits up into a union of subsets from $D_{1}$ and $D_{2}$, and every such union gives rise to a subset $A$, we see that

$$
\begin{aligned}
\sum_{A \subseteq E(D)} t^{r(D)-r(A)} z^{|A|-r(A)}= & \\
& \left(\sum_{A_{1} \subseteq E\left(D_{1}\right)} t^{r\left(D_{1}\right)-r\left(A_{1}\right)} z^{\left|A_{1}\right|-r\left(A_{1}\right)}\right)\left(\sum_{A_{2} \subseteq E\left(D_{2}\right)} t^{r\left(D_{2}\right)-r\left(A_{2}\right)} z^{\left|A_{2}\right|-r\left(A_{2}\right)}\right) .
\end{aligned}
$$

Hence $f(D)=f\left(D_{1}\right) \cdot f\left(D_{2}\right)$, as desired. 
As usual, deletion of an edge $e$ means erasing it from the digraph and contraction of $e$ means erasing $e$ and identifying the two endpoints of $e$. We will not contract loops. We now prove a proposition showing how $f(D)$ can be computed recursively by contracting and deleting an edge. This is similar to the standard deletion-contraction development of the Tutte polynomial for an ordinary graph (or matroid) [4].

Proposition 2.5. Let $D$ be a rooted digraph, with distinguished vertex *. Let $e$ be an edge emanating from *, i.e., $\{e\}$ is a feasible set. Then

$$
f_{D}(t, z)=f_{D / e}(t, z)+t^{r(D)-r(D-e)} f_{D-e}(t, z) .
$$

Proof. Let $e$ be an edge emanating from *. We know that

$$
f_{D}(t, z)=\sum_{e \in A \subseteq E(D)} t^{r(D)-r(A)} z^{|A|-r(A)}+\sum_{e \notin A \subseteq E(D)} t^{r(D)-r(A)} z^{|A|-r(A)} .
$$

First, assume $e \in A \subseteq E(D)$. There exists a maximal feasible set in $A$ containing $e$; this fact is straightforward. We then see that $r(D / e)=r(D)-$ $1, r(A / e)=r(A)-1$ and $|A / e|=|A|-1$, so since $t^{r(D)-r(A)} z^{|A|-r(A)}=$ $t^{(r(D)-1)-(r(A)-1)} z^{(|A|-1)-(r(A)-1)}$, we have

$$
\sum_{e \in A \subseteq E(D)} t^{r(D)-r(A)} z^{|A|-r(A)}=\sum_{A^{\prime} \subseteq E(D / e)} t^{r(D)-r\left(A^{\prime}\right)} z^{\left|A^{\prime}\right|-r\left(A^{\prime}\right)}=f_{D / e}(t, z) .
$$

Now assume $e \notin A \subseteq E(D)$. Note that if $A^{\prime}$ corresponds to $A$ in the deletion $D-e$, then $|A|=\left|A^{\prime}\right|$ and $r(A)=r\left(A^{\prime}\right)$. Thus,

$$
\begin{aligned}
f_{D-e}(t, z)= & \sum_{A^{\prime} \subseteq E(D-e)} t^{r(D-e)-r\left(A^{\prime}\right)} z^{\left|A^{\prime}\right|-r\left(A^{\prime}\right)}, \quad \text { so } t^{r(D)-r(D-e)} f_{D-e}(t, z)= \\
& \sum_{e \notin A \subseteq E(D)} t^{r(D)-r(A)} z^{|A|-r(A)} .
\end{aligned}
$$

Hence, from equation (1), $f_{D}(t, z)=f_{D / e}(t, z)+t^{r(D)-r(D-e)} f_{D-e}(t, z)$.

We now give an example where we use Proposition 2.5 to compute $f_{D}(t, z)$.

Example 2.6. In Figure 2, the deleted and contracted graphs are placed beneath the parent graph. The computations of $f_{D}$ for the remaining digraphs is routine. We obtain $f_{D}(t, z)=\left(t^{3}+t^{2}+2 t+1\right) z^{2}+\left(2 t^{3}+4 t^{2}+6 t+3\right) z+\left(t^{3}+3 t^{2}+5 t+3\right)$.

We now restrict our attention to rooted arborescences $T$. The following proposition is the first step toward showing that $f_{T}(t, z)$ distinguishes arborescences, but is of interest in its own right.

Proposition 2.7. Let $T$ be a rooted arborescence with distinguished vertex *, and suppose $\operatorname{deg}(*)=1$. Then $f_{T}(t, z)$ is irreducible over $\mathrm{Z}[t, z]$.

Proof. Assume $T$ is a rooted arborescence, and $\operatorname{deg}(*)=1$. Let $e$ be the one edge adjacent to $*$. From Proposition 2.4, $f_{T}(t, z)=f_{T / e}(t, z)+$ $t^{r(T)-r(T-e)} f_{T-e}(t, z)$. Note first that $T-e$ has no edge connected to *, so 


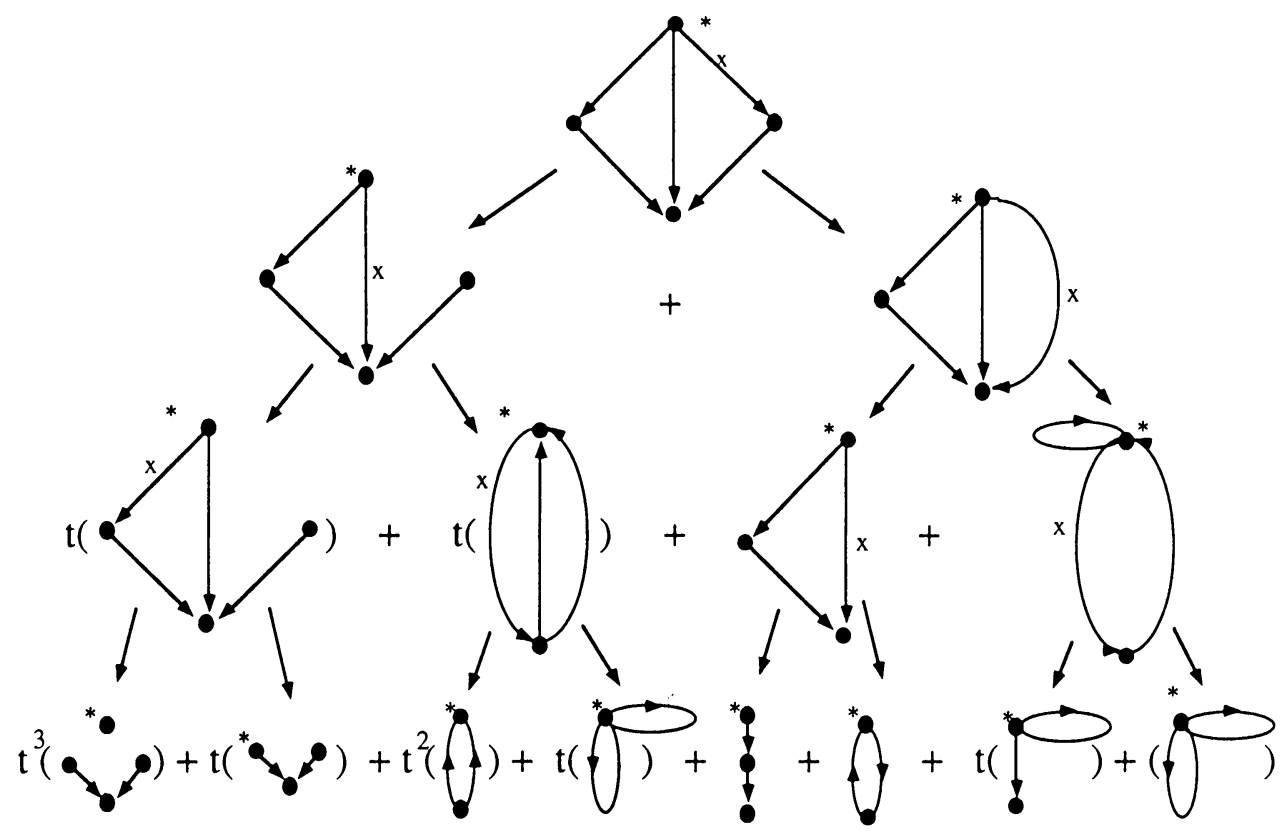

FIGURE 2

$T-e$ contains no feasible sets, hence $r\left(A^{\prime}\right)=0$, for every subset $A^{\prime}$ of edges of $T-e$. Let $n=|E(T)|$. Then $r(T)=n$, so we have $f_{T}(t, z)=$ $f_{T / e}(t, z)+t^{n} f_{T-e}(t, z)$.

Now,

$f_{T-e}(t, z)=\sum_{A^{\prime} \subseteq E(T-e)} t^{r(T-e)-r\left(A^{\prime}\right)} z^{\left|A^{\prime}\right|-r\left(A^{\prime}\right)}=\sum_{A^{\prime} \subseteq E(T-e)} z^{\left|A^{\prime}\right|}=(z+1)^{n-1}$.

Thus, $f_{T}(t, z)=f_{T / e}(t, z)+t^{n}(z+1)^{n-1}$.

Next, in $f_{T / e}(t, z)$, the highest power of $t$ that appears is $r(T / e)=n-1$; the highest power of $z$ that could appear is at most $n-2$, by Lemma $2.1 \mathrm{f}$. Thus, $f_{T}(t, z)=t^{n} z^{n-1}+f_{1}(t, z)$, where the largest power of $z$ that appears in $f_{1}(t, z)$ is at most $n-2$.

Now, suppose $f_{T}(t, z)=g(t, z) h(t, z)$ is a factorization of $f_{T}(t, z)$ over $\mathrm{Z}[t, z]$. Write $g(t, z)=t^{r} z^{s}+g_{1}(t, z)$, where $s$ is the largest power of $z$ that appears throughout $g$, and $r$ is the largest power of $t$ that appears among terms containing $z^{s}$. In a similar fashion, write $h(t, z)=t^{u} z^{v}+h_{1}(t, z)$, with $v$ the largest power of $z$ and $u$ the largest power of $t$, given $v$. Since all exponents 
that appear are greater than or equal to 0 , we have $s+v=n-1$ and $r+u=n$, and (WLOG) $r \leq s$ (if both $r>s$ and $u>v$, then $n=r+u \geq s+v+2=n+1$ ). Thus, in $g(t, z)$, there is at least one term where the $z$ exponent is greater than or equal to the $t$ exponent; first, select the terms where the $z$ exponent exceeds the $t$ exponent by the greatest amount (possibly zero), then, among all such terms, choose $t^{a} z^{b}$ with $a+b$ maximal.

Now, $f_{T}(t, z)$ has a constant term, corresponding to $A=T$, hence $h(t, z)$ has a constant term, in particular, a term where the $z$ exponent is equal to the $t$ exponent. So as above in $g(t, z)$, select all terms where the $z$ exponent exceeds the $t$ exponent by the most (possibly zero) in $h(t, z)$, and then among those, pick out $t^{c} z^{d}$ where $c+d$ is maximal. Now, in $g(t, z) h(t, z), m t^{a+c} z^{b+d}$ cannot be cancelled by any other term or we violate the choice of $a, b, c$ and $d$. Hence, we have $b+d \geq a+c$. However, by Lemma $2.1 f, b+d \leq a+c$, so $b+d=a+c$, and again by the same Lemma, $b+d=a+c=0$, whence $a=b=c=d=0$. Thus, $r=s=0$, so $g(t, z)= \pm 1$, by choice of $r$ and $s$. Thus, the factorization of $f_{T}(t, z)$ is trivial, so $f_{T}(t, z)$ is irreducible, as desired.

We can now prove the main theorem of this section.

Theorem 2.8. Let $T_{1}$ and $T_{2}$ be rooted arborescences. If $f\left(T_{1}\right)=f\left(T_{2}\right)$, then $T_{1} \cong T_{2}$.

Proof. Let $T_{1}$ and $T_{2}$ be rooted arborescences with distinguished vertices $*_{1}$ and $*_{2}$, respectively, such that $f\left(T_{1}\right)=f\left(T_{2}\right)$. Since $|E(T)|$ can be determined from $f(T)$, we may assume that $\left|E\left(T_{1}\right)\right|=\left|E\left(T_{2}\right)\right|=n$. We will prove the result by induction on $n$.

If $n=1$, then for $i=1,2, T_{i}$ has one edge emanating from $*_{i}$, so $T_{1} \cong T_{2}$.

Now let $n>1$. Assume we have arborescences $T_{1}$ and $T_{2}$ with $f\left(T_{1}\right)=$ $f\left(T_{2}\right)$. Since $\operatorname{deg}\left(*_{i}\right)$ can also be determined from $f\left(T_{i}\right)$ by Lemma 2.1.a, we let $k=\operatorname{deg}\left(*_{i}\right)$.

Case 1. $k=1$. Let $e_{i}$ be the edge emanating from $*_{i}$ for $i=1,2$ (so $T_{1}$ and $T_{2}$ look as in Figure 3). From Proposition 2.5, $f\left(T_{i}\right)=f\left(T_{i} / e_{i}\right)+$ $t^{r\left(T_{i}\right)-r\left(T_{i}-e_{i}\right)} f\left(T_{i}-e_{i}\right)$, and, as in the proof of the previous proposition, $f\left(T_{i}\right)=$ $f\left(T_{i} / e_{i}\right)+t^{n}(z+1)^{n-1}$. Hence, $f\left(T_{1} / e_{1}\right)=f\left(T_{2} / e_{2}\right)$. By induction, since each $T_{i} / e_{i}$ has $n-1$ edges, we have $T_{1} / e_{1} \cong T_{2} / e_{2}$. However, since $\operatorname{deg}\left(*_{i}\right)=1$, $T_{i}$ is uniquely determined by $T_{i} / e_{i}$ (see Figure 3 ). Hence $T_{1} \cong T_{2}$, and we have the result.

Case 2. $k>1$. Each $T_{i}$ is the direct sum of $k$ components, $T_{i, 1}, \ldots, T_{i, k}$, one for each edge emanating from $*_{i}$. By Proposition 2.4, for $i=1,2$,

$$
f\left(T_{i}\right)=\prod_{j=1}^{k} f\left(T_{i, j}\right) .
$$




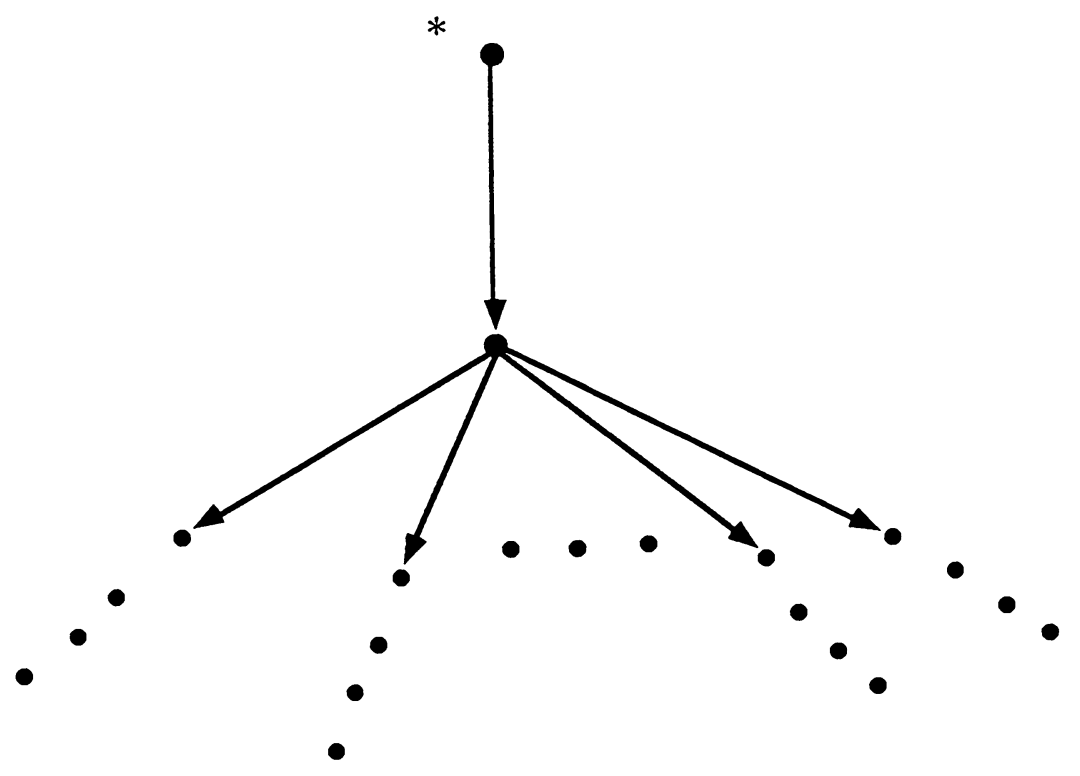

FiguRe 3

By Proposition 2.7, each $f\left(T_{i, j}\right)$ is irreducible. Since $\mathbf{Z}[t, z]$ is a unique factorization domain,

$$
\prod_{j=1}^{k} f\left(T_{1, j}\right)=\prod_{j=1}^{k} f\left(T_{2, j}\right)
$$

implies that, after renumbering, $f\left(T_{1, j}\right)=f\left(T_{2, j}\right)$ for all $j$. But then, by induction, $T_{1, j} \cong T_{2, j}$, since each $T_{i, j}$ must have fewer than $n$ edges (as $k>1)$. Thus, $T_{1} \cong T_{2}$, and we are done.

\section{GENERALIZATION TO GREEDOIDS AND EXAMPLES}

As noted above, several of the ideas developed for rooted digraphs carry over directly to the more general greedoid structure. We begin this section by relating the polynomial $f_{G}(t, z)$ defined on a greedoid $G$ to the one variable polynomial $\lambda_{G}(t)$ defined in [1, Section 6]. We will need to define deletion and contraction for greedoids.

Definition 3.1. Let $G=(E, F)$ be a greedoid, with $A \subseteq E$. Define the deletion $G-A=\left(E-A, F_{1}\right)$ by specifying the feasible sets $F_{1}=\{\mathbf{F} \subseteq E-A: \mathbf{F} \in F\}$ and, if $A$ is feasible, define the contraction $G / A=\left(E-A, F_{2}\right)$ by $F_{2}=\{\mathbf{F} \subseteq$ $E-A: \mathrm{F} \cup A \in F\}$. Thus $G-A$ and $G / A$ are both greedoids on the ground set $E-A$. The reader can check that these definitions correspond to the intuitive ideas of deletion and contraction given in Section 2 when $G$ is a rooted digraph.

We now state the greedoid version of Proposition 2.5, the proof of which is essentially the same as before. 
Proposition 3.2. Let $G=(E, F)$ be a greedoid and let $\{e\}$ be a feasible set. Then $f_{G}(t, z)=f_{G / e}(t, z)+t^{r(G)-r(G-e)} f_{G-e}(t, z)$.

Since every greedoid of positive rank must have a feasible singleton, this proposition gives a recursive way to compute $f_{G}(t, z)$. On the other hand, if $r(G)=0$, then it is easy to see $f_{G}(t, z)=(z+1)^{n}$, where $n=|E|$.

The greedoid polynomial $\lambda_{G}(t)$ is usually defined in terms of basis activities. Although the definition given below depends on an ordering of the elements of $E$, the polynomial is independent of the particular ordering. If $\operatorname{ext}(B)$ is the set of elements of $E$ externally active in a basis $B$ for some given ordering $O$ (that is, $x$ is externally active in $B$ if $B<(B \cup x)-y$ for all $y$ in $B$ such that $(B \cup x)-y$ is a basis) and $B_{G}$ is the collection of all bases, then

$$
\lambda_{G}(t)=\sum_{B \in B_{G}} t^{|\operatorname{ext}(B)|}
$$

Finally, recall $e$ is a coloop in $G$ iff $e$ is in every basis of $G$. Again, more details can be found in [1] or [2].

Theorem 3.3. Let $G=(E, F)$ be a greedoid, with $|E|=n$. Then $\lambda_{G}(t)=$ $f_{G}(0, t-1)$.

Proof. First note that if $r(G)=0$, then $\lambda_{G}(t)=t^{n}$ and $f_{G}(t, z)=(z+1)^{n}$, so the theorem is true in this case. Next, assume $r(G)>0$. Let $\{e\}$ be a feasible set. We will show $f_{G}(t, z)$ satisfies the same recursion as $\lambda_{G}(t)$ (see [2, Theorem 9.6.2]).

Case 1. $e$ is a coloop in $G$. Then $r(G)>r(G-e)$, so $f_{G}(0, t-1)=$ $f_{G / e}(0, t-1)$ by Proposition 3.2.

Case 2. $e$ is not a coloop in $G$. Then $r(G)=r(G-e)$, so again by 3.2, $f_{G}(0, t-1)=f_{G / e}(0, t-1)+f_{G-e}(0, t-1)$.

But this is the same recursion from the reference mentioned above; since $f_{G}(0, t-1)$ and $\lambda_{G}(t)$ agree on trivial greedoids, we are done.

The following proposition follows immediately from the corank-nullity development for the Tutte polynomial of a matroid [4] and the definition of $f_{G}$.

Proposition 3.4. If $G=(E, F)$ is a matroid, then $T_{G}(x, y)=f_{G}(x-1, y-1)$, where $T_{G}(x, y)$ is the Tutte polynomial of $G$.

It is also worth interpreting the recursion given by 3.2 in the case when $G$ is a matroid. In this case, $\{e\}$ is feasible if and only if $e$ is not a loop. If $e$ is not an isthmus (coloop), then $r(G)=r(G-e)$, so 3.2 gives $f_{G}=f_{G / e}+f_{G-e}$. If $e$ is an isthmus, we have (by convention) $G-e=G / e, r(G)=r(G / e)+1$, and $t=x-1$, so 3.2 yields $f_{G}=f_{G / e}+(x-1) f_{G-e}=x f_{G / e}$. Finally, since $z=y-1$, we have $f_{G}=y^{n}$ where $r(G)=0$ and $|E|=n$, i.e., $G$ consists of $n$ loops. These recursions are (essentially) the standard Tutte deletion-contraction recursion for matroids. See [4] or [7], for example. 
It is also easy to generalize Lemmas 2.1, 2.2 and Proposition 2.4 to the greedoid case. We state these generalizations here for completeness. Recall that a greedoid $G$ is full if $r(G)=|E(G)|$, i.e., $E$ is a feasible set.

Lemma 3.5. Let $G$ be a greedoid.

a. The coefficient of $t^{r-1}$ is the number of feasible singletons, where $r=r(D)$.

b. $f_{G}(1,1)=2^{|E(G)|}$.

c. $f_{G}(1,0)=$ the number of feasible sets.

d. $f_{G}(0,1)=$ the number of spanning sets.

e. $f_{G}(0,0)=$ the number of bases.

f. If $G$ is a full greedoid, then for any term $c t^{a} z^{b}$ in $f_{G}, a \geq b$. Further, $a=b$ implies $a=b=0$.

Lemma 3.6. The following are equivalent:

1. $G$ is a full greedoid.

2. $f_{G}(0,1)=1$.

3. In every term the $z$ exponent $\leq$ the $t$ exponent.

4. There is no pure $z$ term.

The (unordered) direct sum of two greedoids is defined in precisely the same way the direct sum of two rooted digraphs was defined. That is, the feasible sets of $G_{1} \oplus G_{2}$ are precisely the disjoint unions of the feasible sets of $G_{1}$ and $G_{2}$. The proof of the next proposition is essentially the same as the proof of 2.4 .

Proposition 3.7. If $G=G_{1} \oplus G_{2}$, then $f(G)=f\left(G_{1}\right) \cdot f\left(G_{2}\right)$.

As with matroids, a special case of this proposition describes the behaviour of $f(G)$ for loops and isthmuses. In a greedoid $G$, an element $e$ is a loop if it is in no feasible set, and $e$ is an isthmus if $F$ is feasible precisely when $F \cup\{e\}$ is feasible (i.e., $e$ can be added to or deleted from any feasible set). Then, if $e$ is a loop, we get $f(G)=(z+1) \cdot f(G-e)$, and, when $e$ is an isthmus $f(G)=(t+1) \cdot f(G / e)$.

We now give two counterexamples to show Theorem 2.8 cannot be extended to rooted digraphs which are not arborescences (Example 3.8) or to full greedoids (Example 3.9). Example 3.9 also shows $f_{G}$ does not distinguish the class of rooted arborescences.

Example 3.8. Let $D_{1}$ and $D_{2}$ be the two digraphs of Figure 4. Then the reader may easily verify that $f\left(D_{1}\right)=f\left(D_{2}\right)=(z+1)\left(t^{2} z+t^{2}+t+1\right)$, but $D_{1}$ and $D_{2}$ are clearly not isomorphic.

Example 3.9. Let $E=\{a, b, c\}$ and define full greedoids $G_{1}=\left(E, F_{1}\right)$ and $G_{2}=\left(E, F_{2}\right)$ with feasible sets given by $F_{1}=\{\varnothing,\{a\},\{b\},\{a, b\},\{a, c\}$, $\{a, b, c\}\}$ and $F_{2}=\{\varnothing,\{a\},\{b\},\{a, c\},\{b, c\},\{a, b, c\}\} . G_{1}$ is the directed branching greedoid (rooted digraph) of Figure 5. Again, the reader can check $f\left(G_{1}\right)=f\left(G_{2}\right)=\left(t^{3}+t^{2}\right) z+\left(t^{3}+2 t^{2}+2 t+1\right)=(t+1)\left(t^{2} z+t^{2}+t+1\right)$. 


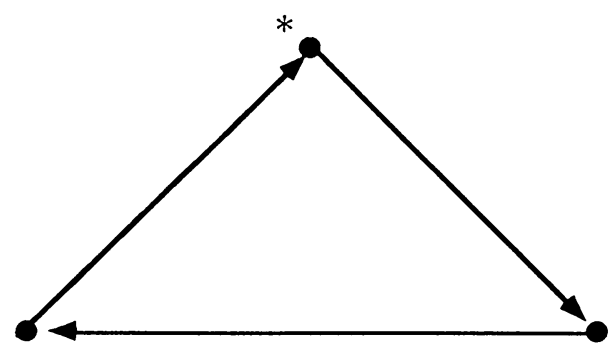

$\mathrm{D}_{1}$

\section{FigURE 4}

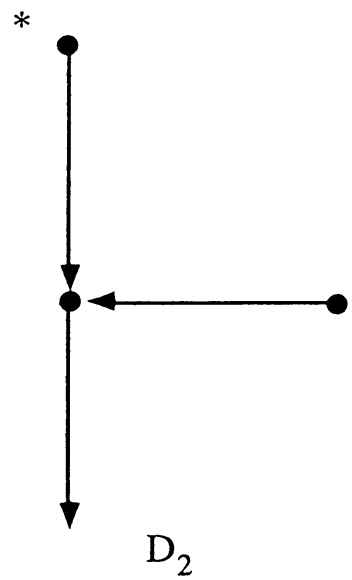

However, it is easy to show that $G_{2}$ cannot be isomorphic to a rooted digraph, so $G_{1}$ and $G_{2}$ are not isomorphic. Since $G_{1}$ is a rooted arborescence, this also shows $f(G)$ is unable to distinguish rooted arborescences within the class of all greedoids (or even full greedoids, by 3.6). We also note that although $f\left(G_{2}\right)$ factors, $G_{2}$ cannot be expressed as a direct sum of non-trivial greedoids. Thus, the converse to 3.7 is false.

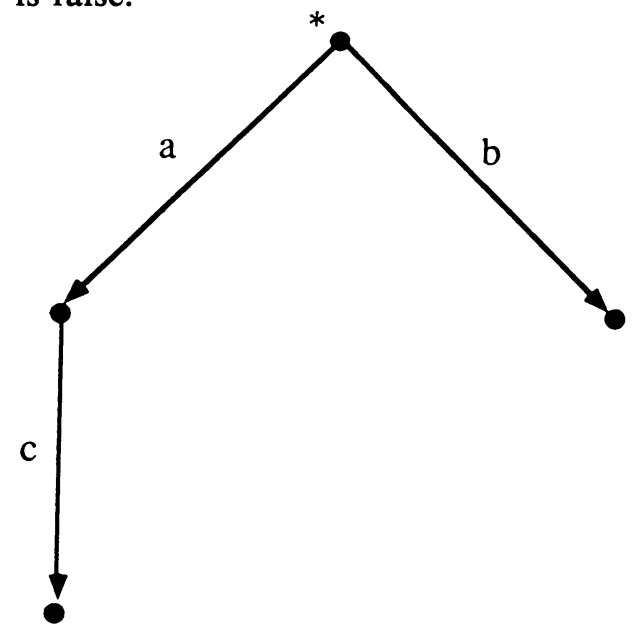

Figure 5

Remark 3.10. The definition of an isthmus in a greedoid, as given in the discussion following 3.7, is not uniformly accepted. In [2], for example, a coloop is defined as an element $e$ which is in every basis of the greedoid. Thus, for full greedoids (in particular, rooted arborescences), every edge is a coloop. Our definition is motivated by the direct sum properties of isthmuses in matroid theory. Using our definition, an edge in a rooted digraph is an isthmus if and only if it emanates from $*$ and its terminal vertex has out-degree zero. For example, in Figure 5, while every edge is a coloop, only $b$ is an isthmus. 
Remark 3.11. If we define $g(G)=t^{-r(G)} f(G)$, then we can rewrite the recursion given in 3.2 as $g(G)=t^{-1} g(G / e)+g(G-e)$. This follows from the fact that $r(G / e)=r(G)-1$ when $\{e\}$ is a feasible set. We also note that 3.7 holds for $g(G)$, i.e., if $G=G_{1} \oplus G_{2}$, then $g(G)=g\left(G_{1}\right) \cdot g\left(G_{2}\right)$.

Remark 3.12. Since there is a one-to-one correspondence between rooted directed arborescences and rooted undirected arborescences, Proposition 2.7 and Theorem 2.8 hold for rooted (undirected) graphs. The other results in Section 2 can also be translated to the undirected case. We leave the details to the reader.

We conclude with some conjectures and problems.

1. Show that Theorem 2.8 is true for a larger class of rooted digraphs, e.g. for $\{D$ : every edge of $D$ is in some feasible set $\}$.

2. By Theorem 2.8, $f(T)$ determines $T$ when $T$ is a rooted arborescence, thus $T$ can be reconstructed from $f(T)$ in this case. In fact, the proof of the theorem gives a recursive algorithm for doing this. It would be interesting to construct other algorithms for reconstruction.

\section{ACKNOWLEDGMENT}

We wish to thank Lorenzo Traldi for useful suggestions and comments.

\section{REFERENCES}

1. A. Bjorner, B. Korte and L. Lovasz, Homotopy properties of greedoids, Advances in Applied Mathematics, 6 (1985), 447-494.

2. A. Bjorner and G. Ziegler, Introduction to greedoids, preprint (1987) to appear in: N. White, ed., Combinatorial geometries, advanced theory, Cambridge Univ. Press, Cambridge.

3. T. Brylawski and D. Kelly, Matroids and combinatorial geometries, Lecture Notes Series, UNC Press, Chapel Hill, NC, 1980.

4. T. Brylawski and J. Oxley, The Tutte polynomial, preprint (1987) to appear in: N. White, ed., Combinatorial geometries, advanced theory, Cambridge Univ. Press, Cambridge.

5. G. Gordon and L. Traldi, Polynomials for directed graphs, submitted.

6. B. Korte and L. Lovasz, Polymatroid greedoids, Journal of Combinatorial Theory, Series B 38 (1984), 41-72.

7. W. Tutte, Graph theory, Cambridge Univ. Press, Cambridge, 1984.

8. N. White, ed., Matroid Theory, Cambridge Univ. Press, Cambridge, 1985.

(Address of both authors): Department of Mathematics, Lafayette College, Easton, Pennsylvania 18042 